\title{
Epidemiologia da Esquistossomose Mansônica em Área de Baixa Endemicidade ${ }^{1}$
}

\section{Epidemiology of Schistosomiasis Mansoni in a Low Endemic Area}

\author{
Luis Candido de S. Dias'; Carmem M. Glasser ${ }^{3}$; \\ Oswaldo Marçal Jr. ${ }^{4}$ \& Patrícia Ivana P. Bonesso ${ }^{2}$
}

\begin{abstract}
DIAS, L. C. S.; GLASSER, C. M.; MARÇAL fr., O. E BONESSO, P. I. P. Epidemiology of Schistosomiasis Mansoni in a Low Endemic Area. Cad. Saúde Públ., Rio de Faneiro, 10 (supplement 2): 254-260, 1994.

We discuss the epidemiological patterns of schistosomiasis mansoni in areas with low transmission in Brazil. We define as areas of low endemicity those where the prevalence is less than 10\%, the number Schistosoma mansoni eggs per gram of feces (epg) is less than 96, and carriers are asymptomatic. Data are from the county of Pedro de Toledo in the Ribeira Valley (São Paulo State) and were collected randomly according to the aggregate pattern of S. mansoni within the hosts. We suggest the replacement of parasitological methods by more sensitive and specific serological techniques. The main risk factor for infection is type of leisure activity. Infection is more frequent in the 10-14, 15-19, and 20-24-year age brackets. Geometric mean epg is 58.5. Intensity of infections correlates well $(r s=0.745)$ with prevalence. The highest index of potential contamination is in the 5-20-year age bracket (57.6\%). Autochthonous cases show close association with Biomphalaria tenagophila, which has a low infection rate (2\%). Prevalence, incidence, and intensity of infection patterns are similar to those of moderate and high endemic areas. Social and cultural aspects must be studied in order to obtain a global epidemiological view of schistosomiasis.
\end{abstract}

Key words: Schistosomiasis; Schistosoma mansoni; Low Endemicity; Epidemiology

\section{INTRODUÇÃO}

A epidemiologia da esquistossomose em suas linhas gerais é bem conhecida. Ela não é, necessariamente, uniforme dentro de um país endêmico e é quaseimpossível de ser comparada entre países. Sua epidemiologia é tão variada

\footnotetext{
${ }^{1}$ Pesquisa financiada pela Fundação de Amparo à Pesquisa do Estado de São Paulo (Fapesp), Superintendência de Controle de Endemias (Sucen) e Conselho Nacional de Desenvolvimento Científico e Tecnológico $(\mathrm{CNP} q)$.

2 Instituto de Biologia da Universidade Estadual de Campinas. Caixa Postal 6109, Campinas, SP, 13083-970, Brasil.

${ }^{3}$ Superintendência de Controle de Endemias. Rua Paula Souza, 166, São Paulo, SP, 01027-000, Brasil.

${ }^{4}$ Departamento de Biociências do Centro de Ciências Biomédicas da Universidade Federal de Uberlândia. Av. Foão Pinheiro, 565, Campus Umuarama, Caixa Postal 593, Uberlândia, MG, 384000-902, Brasil.
}

quanto a ecologia humana e o ambiente no qual a esquistossomose ocorre. Os parâmetros epidemiológicos, como prevalência, incidência, intensidade de infecção e morbidade, variam, amplamente, mesmo dentro de uma região. Essa diversidade pode ser explicada, principalmente, pela ecologia humana e o ambiente (Doumenge et al., 1987)

É tarefa difícil propor parâmetros para avaliar o grau de endemicidade da esquistossomose mansônica devido a seu caráter focal e sua ampla diversidade. Todavia, pode tentar uma classificação. Uma área seria considerada de alta endemicidade quando houvesse altas prevalência e intensidade de infecção, geralmente, em crianças entre 5 e 15 anos de idade e formas crônicas em adultos. Nas áreas de moderada ou baixa endemicidade, a distribuição geográfica dos portadores e da morbidade severa estaria bem localizada, em focos nitida- 
mente delimitados. Esse aspecto pode ainda ser verificado em área de alta transmissão, onde foram aplicadas medidas de controle. Apesar da ampla distribuição dos moluscos e as freqüentes oportunidades do contato humano com a água, as altas taxas de transmissão só devem ocorrer em poucos locais (WHO, 1993). O número de ovos por grama de fezes que fornece a intensidade de infecção deve ser considerado ao se tentar classificar os níveis de endemicidade.

Pelo exposto, nota-se que, na tentativa de enquadrar uma área em um nível endêmico, vários aspectos merecem avaliação criteriosa.

Com as devidas ressalvas, poderíamos considerar regiões de média e alta endemicidade aquelas com prevalência superior a $10 \%$, com mais de 120 ovos por grama de fezes e presença de indivíduos com quadro clínico da esquistossomose. Área de baixa endemicidade seria aquela com prevalência inferior a $10 \%$, com a maioria dos infectados assintomáticos e eliminando menos de 96 ovos por grama de fezes (Katz, 1986; WHO, 1985).

A distribuição da esquistossomose hoje no Brasil (Doumenge et al., 1987) não é muito diferente da constatada há 20 anos (Freitas, 1972). As áreas de média e alta endemicidades constituem uma região contínua, predominando no litoral nordestino, do Ceará até a Bahia e em Minas Gerais. No restante a transmissão ocorre em áreas focais, não constituindo um contínuo, sendo eminentemente localizada e de baixa intensidade, com algumas áreas reduzidas de transmissão mais intensa (Silveira, 1989).

A maioria dos trabalhos sobre dinâmica de transmissão da esquistossomose foi realizada em áreas hiperendêmicas, não podendo seus resultados serem diretamente transpostos para áreas de baixa endemicidade. Em anos recentes, a compreensão da dinâmica da transmissão da esquistossomose sofreu considerável avanço (WHO, 1985; Lima e Costa et al., 1985; Warren, 1987; Mott, 1989), devido, principalmente, à ênfase dada à quimioterapia nos programas de controle.

\section{QUESTÕES METODOLÓGICAS}

A epidemiologia da endemia pode ser abordada de várias maneiras. Dificilmente, iremos nos afastar da visão ecológica, uma vez que a presença do hospedeiro intermediário é fator fundamental na presença e distribuição da esquistossomose, assim como a maneira do homem interagir com as coleções líquidas.

$\mathrm{Na}$ dinâmica de transmissão não podemos analisar em partes a relação hospedeiro-parasito. $\mathrm{O}$ padrão de contato humano com as coleções líquidas, a população de hospedeiros intermediários e a intensidade de infecção criam condições de manter um ciclo crescente da infecção. $\mathrm{O}$ conceito de agregação em infeções por helmintos é hoje bem conhecido (Li \& Hsu, 1951). A distribuição dos vermes em populações humanas faz-se de maneira agregada, em que um pequeno número de indivíduos alberga a maioria dos parasitos, eliminando, assim, a maioria dos ovos. O restante da população está infectada por um pequeno número de vermes. $\mathrm{O}$ fenômeno de agregação idealizado por Bradley (Li \& Hsu, 1951; Bradley, 1972; Bradley \& May, 1978) constitui a base para melhor estudar a epidemiologia e aplicar as medidas mais adequadas de controle da esquistossomose. A transmissão da parasitose é melhor explicada por agregados, como ocorre nas áreas de alta endemicidade (Bradley, 1972; Cohen, 1977; Bradley \& May, 1978; Polderman, 1979; Woolhouse, 1991; Jordan \& Webbe, 1993). Desafortunadamente, os modelos matemáticos e sua correspondente demonstração empírica não são adequados às situações de baixa transmissão (Silva, 1992). No Estado de São Paulo, localizado em área de baixa endemicidade, o comportamento da dinâmica de transmissão é pouco estudado. Há algumas diferenças com as áreas de média e alta transmissão que tentaremos expor a seguir.

A ocorrência e distribuição da esquistossomose no Estado de São Paulo não teria um determinante predominante, mas um conjunto de fatores que, em cada área de transmissão, apresentaria importância relativa maior ou menor. Lazer, migração, antigüidade dos focos, atividades profissionais na rizicultura e na horticultura, são alguns fatores que, em conjunto, estabeleceriam os padrões de transmissão no território paulista. Mais especificamente, na região de Itariri e Pedro de Toledo, no vale do rio Ribeira de Iguape, onde estamos trabalhando desde 1980, um conjunto de fatores agiu para favorecer a transmissão. Esses fatores seriam o 
clima úmido e quente e a existência de uma atividade sócio-ecônomica propícia. Exceto essas duas áreas, a transmissão no Estado de São Paulo parece ser hoje casual, ocorrendo nas áreas de maior densidade dos hospedeiros intermediários, mas sem fatores comuns que determinem o contato com as coleções líquidas. Em alguns focos, o lazer seria fator de destaque.

Ao nos depararmos com uma área na qual a esquistossomose é de baixa transmissão ou o controle foi bem-sucedido, há necessidade de amostrá-la aleatoriamente para estudos epidemiológicos, lembrando do fenômeno de agregação dos casos (Marçal Jr. et al., 1993).

Uma vez amostrada a população, cabe-nos escolher o método laboratorial mais adequado. No nível individual, o diagnóstico em laboratório não apresenta maiores dificuldades. Ao realizarmos inquéritos populacionais da helmintíase, onde ocorre níveis de média e alta endemicidade, os métodos parasitológicos e, principalmente, a técnica de Kato-Katz possuem adequada sensibilidade. Todavia, os métodos parasitológicos em áreas de baixa transmissão ou sob controle subestimam os resultados, levando nos a conclusões errôneas (HoshinoShimizu et al., 1986; Dias et al., 1992; Hoshino Shimizu et al., 1992; Savigny, 1992). Nesse caso, os testes imunológicos são úteis, sendo indicados a imunofluorescência indireta, o teste imunoenzimático (Elisa), a reação periovular e os testes radioimunológicos que detectam anticorpos circulantes (Mott \& Dixon, 1982). Há ainda reações imunológicas baseadas em antígenos (Ruppel et al., 1990; Jonge, 1992; Ripert et al., 1992). Assim, a seleção adequada de um teste para estudos soroepidemiológicos deve obedecer a alguns critérios, tais como ser sensível e específico; prático e ecônomico no campo; ter resultados de fácil interpretação; os reagentes usados no teste devem ser estáveis, permitindo reproduzir os resultados; as amostras de sangue devem ser coletadas por meio de procedimentos simples.

Em nossa experiência com inquéritos soroepidemiológicos em diferentes áreas de baixa endemicidade no Estado de São Paulo, temos observado que os índices de prevalência pelos testes imunológicos são de duas a três vezes mais elevados do que os detectados pelos métodos parasitológicos de fezes, e que essas diferenças se devem principalmente à baixa sensibilidade dos últimos (Dias et al., 1971; Dias et al., 1992). A reação de imunofluorescência, utilizando como antígeno cortes de vermes parafinados, tem apresentado resultados satisfatórios quanto aos graus de sensibilidade e especificidade (Deelder \& Kornelis, 1980), proporcionando metodologia útil para diagnóstico da esquistossomose mansônica.

\section{O CASO DE PEDRO DE TOLEDO}

De todas as regiões de ocorrência de focos ativos de esquistossomose em São Paulo, a área dos municípios de Pedro de Toledo e Itariri, no vale do rio Ribeira de Iguape, está sendo uma das mais pesquisadas por nós, desde 1980 (Dias et al., 1988). Nesse ano, como contraposição ao aumento do índice de prevalência humana em Pedro de Toledo, de $4 \%$, em 1970, para $12 \%$, em 1978, intensificou-se o programa de controle no município, com a inclusão de estudos parasitológicos, epidemiológicos e imunológicos, educação sanitária e manejo do ambiente, por meio de obras de saneamento básico (Dias et al., 1988). Segundo esses autores, todos os portadores da endemia eram assintomáticos. Nessa área, o hospedeiro intermediário do trematódeo é a Biomphalaria tenagophila, com índices de infecção natural inferiores a $2 \%$ (Dias et al., 1989). Esses autores, em 1980, por meio de exames de fezes (método de KatoKatz) examinaram 4.741 indivíduos, havendo $22,8 \%$ com ovos de Schistosoma mansoni (três lâminas por indivíduo, da mesma amostra de fezes); entre eles, 583 tinham sido anteriormente tratados para esquistossomose, e os 4.158 restantes nunca foram medicados para a helmintose; as prevalências no dois grupos foram $31,7 \%$ e $21,6 \%$, respectivamente. Por meio de investigação epidemiológica, constatou-se que $83,6 \%$ dos casos eram autóctones da área estudada. Considerando portadores autóctones e não tratados, foram verificadas taxas de prevalência com grande variação, $5,9 \%$ a $61,2 \%$, em 15 localidades na zona rural de Pedro de Toledo; $28,1 \%$ dos homens estavam infectados, e $14,7 \%$ das mulheres; os grupos etários de 10-14, 15-19 e 20-24 apresentavam índices de 
prevalência superiores aos dos outros grupos de idade. Pelo método de Kato-Katz, a intensidade de infecção entre os portadores autóctones e não tratados foi de 58,5 ovos por grama de fezes (média geométrica). Observou-se que de acordo com os grupos etários, há boa correlação $\left(r_{\mathrm{s}}=0,745\right)$ entre intensidade de infecção e prevalência. Somente $0,4 \%$ de 1.137 moluscos coletados eliminavam cercárias de $S$. mansoni (Dias et al., 1989). Em estudo de campo, em 1987, no mesmo município, Marçal Jr. et al. (1991) verificaram que cerca de $80 \%$ dos portadores apresentavam menos de 100 ovos por grama de fezes e apenas $9,0 \%$ eliminavam mais do que a metade do total de ovos. Os mais altos índices de potencial de contaminação ocorreram nos grupos etários entre 5 e 20 anos (57,6\%). A distribuição geográfica dos portadores demonstrou haver evidente agregação dos casos autóctones, assim como íntima associação entre locais de contato da população com os criadouros de B. tenagophila. Os portadores não estão agregados ao acaso. A partir de um estudo de caso-controle por pareamento (1:1), em Pedro de Toledo, durante 1987, foram determinados os fatores de risco para infecção por S. mansoni (Marçal Jr. et al., 1993). Os principais fatores de risco foram contato com água para nadar, brincar e pescar; vadear (passagem)e más condições de higiene. Desta- cou se que as atividades recreativas foram as principais responsáveis pela transmissão do trematódeo em Pedro de Toledo. Achados semelhantes foram relatados em áreas de média endemicidade no Estado de Minas Gerais (Lima e Costa et al., 1987, 1991).

\section{DISCUSSÃO E CONCLUSÕES}

Ao procedermos a análise epidemiológica em área de baixa transmissão e sob controle, é necessário considerarmos a origem dos casos, ou seja, onde aconteceu a infecção do portador e sua variação temporal (Figura 1). Em Pedro de Toledo, foi constatado que o número de casos importados de outros estados brasileiros tem aumentado, contrapondo queda do número de casos autóctones. É bem conhecido o fato de que muitos focos paulista de esquistossomose surgiram após insistente contaminação ambiental por ovos do parasito de portadores oriundos de outros estados (Silva, 1992). Ainda merecem destaque as oscilações dos índices de prevalência e incidência, no período de 1980 a 1991, em Pedro de Toledo (Figura 2). Explicam-se a queda da prevalência e a manutenção da incidência, ambas em patamares inferiores a 5\%, pela eficiência das medidas de controle (Dias et al., 1992).

FIGURA 1. Classificação Epidemiológica e Coeficientes de Prevalência de Portadores de Schistosoma mansoni, pelo Método de Kato-Katz, em Pedro de Toledo (PT), São Paulo, no Período de 1980 a 1991

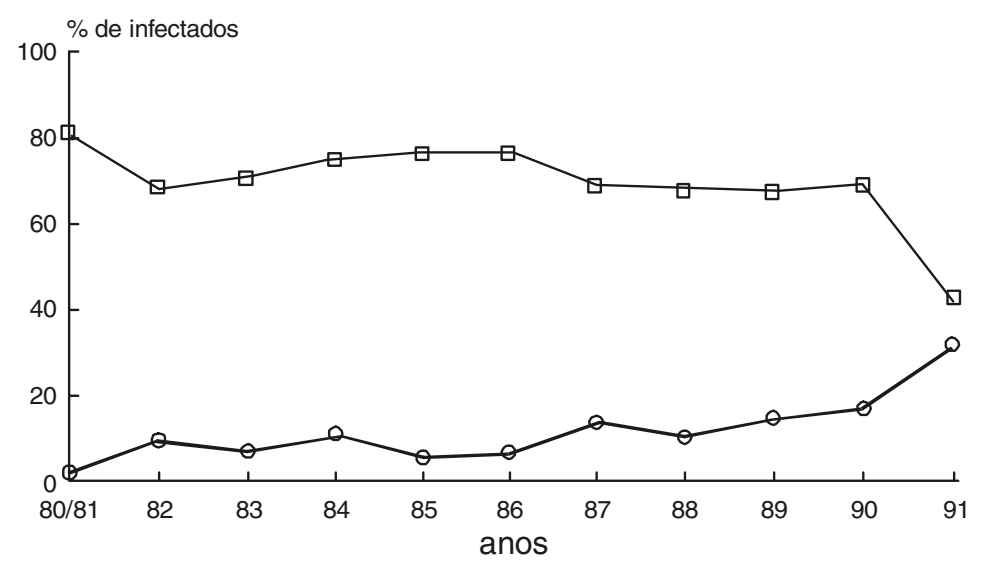

$$
\square \text { Autóctones PT } \quad-\text { Importados }
$$


FIGURA 2. Coeficientes de Prevalência e de Incidência de Portadores de Schistosoma mansoni, pelo Método de Kato-Katz, em Pedro de Toledo, São Paulo, no Período de 1980 a 1991

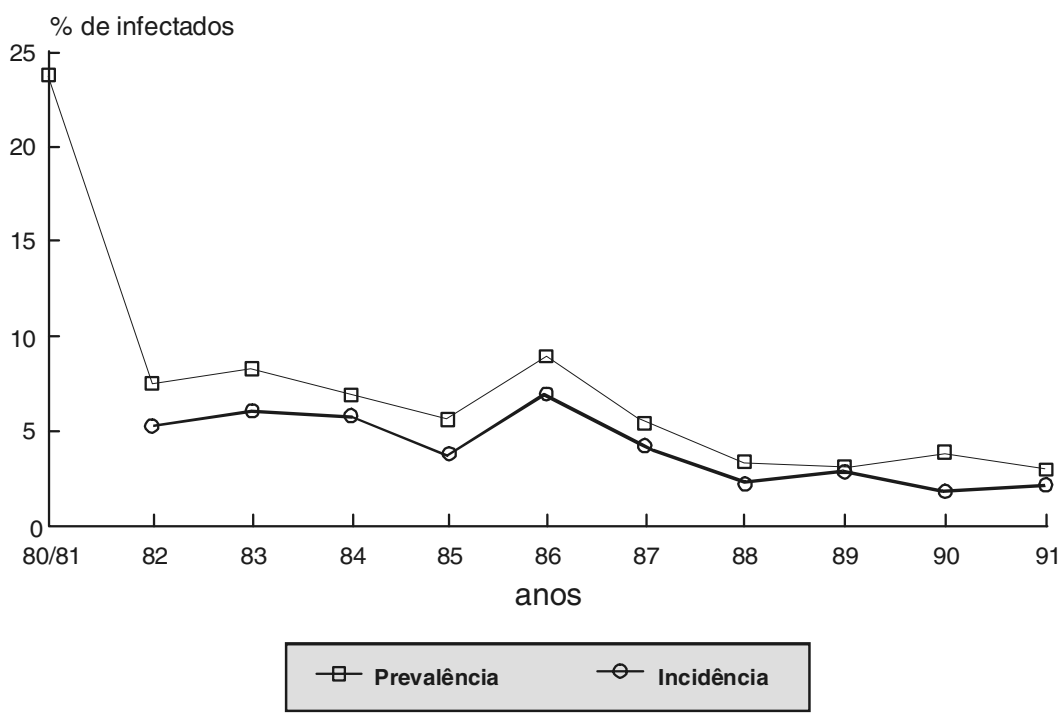

É interessante assinalar que o padrão de prevalência e de intensidade de infecção (Dias et al., 1989) em nossa área de baixa transmissão foi semelhante àquele descrito em áreas com média e alta endemicidade (Coura et al., 1992; Jordan \& Webbe, 1993), ou seja, os picos de intensidade e de prevalência são observados na segunda década de vida. Em seguida, ambas caem, mas a primeira revela queda mais acentuada.

Além da abordagem ecológica que devemos ponderar em áreas de baixa transmissão, é necessário trabalhar nas questões sócio-culturais, permitindo, assim, visão mais global da epidemiologia da esquistossomose mansônica.

Pelo exposto, nota-se que a epidemiologia da esquistossomose em área de baixa endemicidade possui suas peculiaridades. Todavia, de maneira geral, demonstra padrões semelhantes àqueles de regiões com moderada e alta transmissão.

\section{RESUMO}

DIAS, L. C. S.; GLASSER, C. M.; MARÇAL Jr., O. \& BONESSO, P. I. P. Epidemiologia da Esquistossomose Mansônica em Área de Baixa Endemicidade. Cad. Saúde Públ., Rio de Janeiro, 10 (suplemento 2): 254-260, 1994.

Discutem se padrões epidemiológicos da esquistossomos mansônica em áreas brasileiras de baixa endemicidade que possuem prevalência inferior a $10 \%$, menos de 96 ovos por grama de fezes (epg) e onde os infectados são assintomáticos.

Apresentam-se dados do município de Pedro de Toledo (Vale do Ribeira, SP) área de baixa endemicidade cujos resultados foram obtidos de amostragem aleatória devida à agregação de Schistosoma mansoni. Sugere-se substituição de métodos parasitológicos por técnicas sorológicas com maiores sensibilidade e especificidade. O principal fator de risco é o lazer. A infecção predomina nos grupos etários de 10-14, 15-19 e 20-24. A intensidade de infecção foi baixa, com 58,5 epg (média geométrica). Há boa correlação (rs $=0,745)$ entre intensidade de infecção e prevalência. Os mais altos índices de 
potencial de contaminação ocorreram nas idades de 5 a 20 anos (57,6\%). Os casos autóctones mantêm íntimo contato com Biomphalaria tenagophila, com infecção inferior a $2 \%$. Os padrões de prevalência, de incidência e de intensidade de infecção são semelhantes aos de áreas de moderada e alta endemicidade. Questões sócio-culturais merecem estudo para visão global da epidemiologia.

Palavras-Chave: Esquistossomose; Schistosoma Mansoni; Baixa Endemicidade; Epidemiologia

\section{REFERÊNCIAS BIBLIOGRÁFICAS}

BRADLEY, D. J., 1972. Regulation of parasite populations: a general theory of the epidemiology and control of parasitic infection. Transactions of the Royal Society of Tropical Medicine and Hygiene, 66: 697-708.

BRADLEY, D. J. \& MAY, R. M., 1978. Consequence of helminth aggregation for the dynamics of schistosomiasis. Transactions of the Royal Society of Tropical Medicine and Hygiene, 72: 262-273.

COHEN, J. E., 1977. Mathematical models of schistosomiasis. Annual Review of Ecology and Systematics, 8: 209-233.

COURA-FILHO, P.; ROCHA, R. S.; LIMA E COSTA, M. F. \& KATZ, N., 1992. A municipal level approach to the manegement of schistosomiasis control in Peri-Peri, MG, Brazil. Revista do Instituto de Medicina Tropical de São Paulo, 34: 543-548.

DEELDER, A. M. \& KORNELIS, D., 1980. A comparison of the IFA and the ELISA for the demonstration of antibodies against schistosome gut associated polysaccharide antigens in schistosomiasis. Zeitschrift fur Parasitenkund, 64: 65-75.

DIAS, L. C. S.; CAMARGO, M. E.; HOSHINO, S.; RAMOS, A. S.; PIZA, J. T. \& SILVA, L. C., 1971. Inquéritos populacionais da esquistossomose mansoni por técnicas sorológicas de imunofluorescência e de hemaglutinação. Revista do Instituto de Medicina Tropical de São Paulo, 13: 37-44.

DIAS, L. C. S.; GLASSER, C. M.; ETZEL, A.; KAWAZOE, U.; HOSHINO-SHIMIZU, S.; KANAMURA, H. Y.; CORDEIRO, J. A.; MARÇAL Jr., O; CARVALHO, J. F.; GONÇALVES
Jr., F. \& PATUCCI, R., 1988. The epidemiology and control of schistosomiasis mansoni where Biomphalaria tenagophila is the snail host. Revista de Saúde Pública, 22: 462-463.

DIAS, L. C. S.; KAWAZOE, U.; GLASSER, C.; HOSHINO-SHIMIZU, S.; KANAMURA, H. Y.; CORDEIRO, J. A.; GUARITA, O. F. \& ISHIHATA, G. J., 1989. Schistosomiasis mansoni in the municipality of Pedro de Toledo (São Paulo, Brazil) where the Biomphalaria tenagophila is the snail host. I- Prevalence in human population. Revista do Instituto de Medicina Tropical de São Paulo, 31: 110-118.

DIAS, L. C. S.; KANAMURA, H. Y.; HOSHINOSHIMIZU, S.; GLASSER, C. M.; CARVALHO, J. F. \& SILVA, L. C., 1992. Field trials for immunodiagnosis with reference to Schistosoma mansoni. In: Immunodiagnostic Approaches in Schistosomiasis (N. R. Bergquist, ed.), pp. 39-47, Chichester: John Wiley \& Sons.

DOUMENGE, J. P.; MOTT, K. E.; CHEUNG, G.; VILLENAVE, D.; CHAPUIS, O.; PERRINE, $M$. F. \& REAUD THOMAS, G., 1987. Atlas de la Répartition Mondiale des Schistomiases. Talence: CEGETE CNRS/Geneve: OMS \WHO.

FREITAS, C. A., 1972. Situação atual da esquistossomose no Brasil. Revista Brasileira de Malariologia e Doenças Tropicais, 24: 03-63.

HOSHINO-SHIMIZU, S.; CAMARGO, M. E.; KAWADA, H. Y. K.; SILVA, L. C. \& DIAS, L. C. S., 1986. Aspectos sorológicos e soroepidemiológicos da esquistossomose mansônica. In: Modernos Conhecimentos sobre Esquistossomose Mansônica (F. A. Reis \& N. Katz, orgs.), vol. 14, pp. 67-89, Belo Horizonte: Academia Mineira de Medicina. (Suplementos dos Anais de 1983-84 da Academia Mineira de Medicina)

HOSHINO-SHIMIZU, S.; DIAS, L. C. S.; KANAMURA, H. Y.; SILVA, L. C.; GLASSER, C. M. \& PATUCCI, R. M. J., 1992. Soroepidemiology of schistosomiasis mansoni. Memórias do Instituto Oswaldo Cruz, 87 (suppl. IV): 303-306.

JONGE, N., 1992. Detection of the circulating anodic antigen for immunodiagnosis of Schistosoma infections. In: Immunodiagnostic Approaches in Schistosomiasis (N. R. Bergquist, ed.), pp. 111-124, Chichester: John Wiley \& Sons.

JORDAN, P. \& WEBBE, G., 1993. Epidemiology. In: Human Schistosomiasis (P. Jordan, G. Webbe \& R. F. Sturrock, eds.), pp. 87-158, Cambridge: CAB International.

KATZ, N., 1986. Controle da esquistossomose no Estado de Minas Gerais. In: Modernos Conhecimentos sobre Esquistossomose Mansônica (F. A. Reis, I. Faria \& N. Katz, orgs.), vol. 14, pp. 
51-66, Belo Horizonte: Academia Mineira de Medicina. (Suplementos dos Anais de 1983/84 da Academia Mineira de Medicina)

LI, S. Y. \& HSU, H. F., 1951. On the frequency distribution of parasitic helminths in their naturally hosts. Journal of Parasitology, 37: 32-41.

LIMA E COSTA, M. F.; MAGALHÃES, M. A.; ROCHA, R. S.; ANTUNES, C. M. F. \& KATZ, N., 1987. Water-contact patterns and socioeconomic variables in the epidemiology of schistosomiasis mansoni in an endemic area in Brazil. Bulletin of the World Health Organization, 65: 57-66.

LIMA E COSTA, M. F. F.; ROCHA, R. S.; LEITE, M. L. C.; CARNEIRO, R. G.; COLLEY, D.; GAZZINELLI, G. \& KATZ, N., 1991. A multivariate analysis of socio-demographic factors, water contact patterns and Schistosoma mansoni infection in an endemic area in Brazil. Revista do Instituto de Medicina Tropical de São Paulo, 33: 58-63.

LIMA E COSTA, M. F.; ROCHA, R. S.; MAGALHÃES, M. H. A. \& KATZ, N., 1985. A clinico-epidemiological survey of schistosomiasis mansoni in a hyperendemic area in Minas Gerais State (Comercinho, Brazil ). I. Differences in manifestations of schistosomiasis in the town center and in the environs. Transactions of the Royal Society of Tropical Medicine and Hygiene, 79: 539-545.

MARÇAL Jr., O.; HOTTA, L. K.; PATUCCI, R. M. J.; GLASSER, C. M. \& DIAS, L. C. S., 1993. Schistosomiasis mansoni in an area of low transmission. II. Risk factors for infection. Revista do Instituto de Medicina Tropical, 35: 331-335.

MARÇAL Jr., O.; PATUCCI, R. M. J.; DIAS, L. C. S.; HOTTA, L. K. \& ETZELA, A., 1991. Schistosomiasis mansoni in an area of low transmission. I. Impact of control measures. Revista do Instituto de Medicina Tropical de São Paulo, 33: 83-90.

MOTT, K. E., 1989. Contrasts in the control of schistosomiasis. Memórias do Instituto Oswaldo Cruz, 84 (suppl. I): 03-19.
MOTT, E. \& DIXON, H., 1982. Collaborative study on antigen for immunodiagnosis of schistosomiasis. Bulletin of the World Health Organization, 60: 729-753.

POLDERMAN, A. M., 1979. Transmission dynamics of endemic schistosomiasis. Tropical and $\mathrm{Ge}$ ographical Medicine, 31: 465-475.

RIPERT, C.; APRIOU, M. \& TRIBOULEY, J., 1992. Antigen detection in urine samples for the diagnosis of schistosomiasis. In: Immunodiagnostic approaches schistosomiasis (N. R. Bergquist, ed.), pp. 103-109, Chichester: John Wiley \& Sons.

RUPPEL, A.; IDRIS, M. A.; SULAIMAN, S. M. \& HILAI, A. M., 1980. Schistosoma mansoni diagnostic antigens (Sm 31/12): a sero-epidemiological study in Sudan. Tropical Medicine Parasitology, 4: 127-130.

SAVIGNY, D., 1992. Community-based immunodiagnosis of schistosomiasis. In: Immunodiagnostic Approaches in Schistosomiasis (N. R. Bergquist, ed.), pp. 9-27, Chichester: John Wiley \& Sons.

SILVA, L. J., 1992. A Esquistossomose Mansônica no Estado de São Paulo: Origens, Distribuição, Epidemiologia e Controle. Tese de Livre-Docência, São Paulo: Faculdade de Ciências Médicas, Universidade Estadual de Campinas.

SILVEIRA, A. C., 1989. Controle da esquistossomose no Brasil. Memórias do Instituto Oswaldo Cruz, 84 (suppl. I): 91-104.

WARREN, K. S., 1987. Schistosomiasis: past, present and future. Memórias do Instituto Oswaldo Cruz, 82 (suppl. IV): 25-30.

WHO (World Health Organization), 1985. The Control of Schistosomiasis. Geneva: WHO. (Technical Report Series, 728) , 1993. The Control of Schistosomiasis. Geneva: WHO. (Technical Report Series, 830)

WOOLHOUSE, M. G. J., 1991. On the application of mathematical models of schistosomiasis transmission dynamics. I. Natural transmission. Acta Tropica, 49: 241-290. 